\title{
The Bi-Hamiltonian Structure of Fully Supersymmetric Korteweg-de Vries Systems
}

\author{
Walter Oevel ${ }^{1}$ and Ziemowit Popowicz ${ }^{2}$ \\ ${ }^{1}$ Department of Mathematics, University of Paderborn, Warburger Straße 100, \\ W-4790 Paderborn, Federal Republic of Germany \\ ${ }^{2}$ Institute of Theoretical Physics, University of Wroclaw, ul. Cybulskiego 36, \\ PL-50-205 Wroclaw, Poland
}

Received June 11, 1990, in revised form October 2, 1990

\begin{abstract}
The bi-Hamiltonian structure of integrable supersymmetric extensions of the Korteweg-de Vries (KdV) equation related to the $N=1$ and the $N=2$ superconformal algebras is found. It turns out that some of these extensions admit inverse Hamiltonian formulations in terms of presymplectic operators rather than in terms of Poisson tensors. For one extension related to the $N=2$ case additional symmetries are found with bosonic parts that cannot be reduced to symmetries of the classical KdV. They can be explained by a factorization of the corresponding Lax operator. All the bi-Hamiltonian formulations are derived in a systematic way from the Lax operators.
\end{abstract}

\section{Introduction}

Recently there has been much interest in (super-)conformal field theories [1, 2] in context of studying string theories and statistical models of critical phenomena. Along with these studies one observes a growing interest in the supersymmetrization of the Korteweg-de Vries (KdV) equation [3-5]. It turned out that the Virasoro algebra and some of its extensions can be related to the second Hamiltonian structure of the $\mathrm{KdV}$ and $\mathrm{KdV}$-like equations. This Hamiltonian structure is given by a set of Poisson brackets for the fundamental fields representing the Virasoro algebra [6]. In fact, starting from supersymmetric generalizations of the Virasoro algebra and the corresponding Hamiltonian structure it was possible to construct integrable supersymmetric extensions of the classical $\mathrm{KdV}$ equation $[7,8]$.

Related to the $N=1$ superconformal algebra two integrable cases of fermionic extensions of the $\mathrm{KdV}$ equation were found [3,9]. The first case [3] turns out to admit a bi-Hamiltonian formulation, but it fails to be invariant relative to space-supersymmetric transformations. The second case [10] admits such an 
invariance, but it was claimed not to admit a bi-Hamiltonian formulation. We will be interested only in this latter case, it can be written as

$$
\begin{aligned}
\Phi_{t} & =-\Phi^{\prime \prime \prime}+3(\Phi D \Phi)^{\prime} \\
& =\left(-D \partial^{2}+2 \partial \Phi+2 \Phi \partial+D \Phi D\right) \frac{\delta}{\delta \Phi} \int \frac{1}{2} \Phi D \Phi d X,
\end{aligned}
$$

where $\Phi$ is an anticommuting superfunction of the (commuting) space parameter $x$ and one anticommuting Grassmannian variable $\Theta$. The prime indicates differentiation with respect to $x$, the symbol $D$ is to denote the superderivative $D=\Theta \partial+\partial_{\Theta}$ satisfying $D^{2}=\partial \equiv \partial_{x}$. The operator

$$
P_{2}(\Phi):=-D \partial^{2}+2 \partial \Phi+2 \Phi \partial+D \Phi D
$$

in (1.1) is the Hamiltonian operator stemming from the $N=1$ extension of the Virasoro algebra. The (commuting) Hamiltonian function $H:=\int \frac{1}{2} \Phi D \Phi d X$ in (1.1) is obtained by integrating its (anticommuting) density $\frac{1}{2} \Phi D \Phi$ over both space and the Grassmannian $\Theta$, i.e. $d X=d x d \Theta$, where we assume the normalization $\int \Theta d \Theta=1$. Its gradient is understood to be given by the functional derivatives defined through

$$
\left\langle\dot{\Phi}, \frac{\delta H}{\delta \Phi}\right\rangle=\left.\frac{\partial}{\partial \varepsilon}\right|_{\varepsilon=0} H(\Phi+\varepsilon \dot{\Phi}),
$$

with arbitrary vector field $\dot{\Phi}$ and $\langle$,$\rangle representing the duality between vector- and$ co-vector fields.

System (1.1) is integrable in the sense that it admits a Lax formulation with the scattering operator

$$
L=\partial^{2}-\Phi D
$$

(or the gauge equivalent $L=\partial^{2}-D \Phi$ ), i.e. (1.1) is equivalent to $d / d t$ $L=\left[-4\left(L^{3 / 2}\right)_{+}, L\right]$. Here the subscript + denotes the purely differential part of the fractional power of $L$ obtained by formal expansion into pseudo-differential operators (see also Sect. 3).

Expanding the superfunction $\Phi$ into powers of $\Theta$ we may assume $\Phi(x, \Theta)=\xi(x)+\Theta u(x)$ with a bosonic field $u$ and a fermionic field $\xi$. Corresponding to this chart we represent vector fields $K$ and co-vector fields $\gamma$ as

$$
\begin{aligned}
K(\Phi) & =K_{\xi}(u, \xi)+\Theta K_{u}(u, \xi), \\
\gamma(\Phi) & =\gamma_{u}(u, \xi)+\gamma_{\xi}(u, \xi) \Theta,
\end{aligned}
$$

such that the duality between vector and co-vector fields becomes the usual

$$
\langle K, \gamma\rangle=\int K \gamma d x d \Theta=\int\left(K_{u} \gamma_{u}+K_{\xi} \gamma_{\xi}\right) d x .
$$

Regarding $P_{2}(\Phi)$ as a linear map from the co-vector fields to the vector fields the Hamiltonian system (1.1) now can be decomposed into

$$
\begin{aligned}
& \frac{d}{d t}\left(\begin{array}{l}
u \\
\xi
\end{array}\right)=\left(\begin{array}{l}
-u^{\prime \prime \prime}+6 u u^{\prime}-3 \xi \xi^{\prime \prime} \\
-\xi^{\prime \prime \prime}+3(u \xi)^{\prime}
\end{array}\right)
\end{aligned}
$$

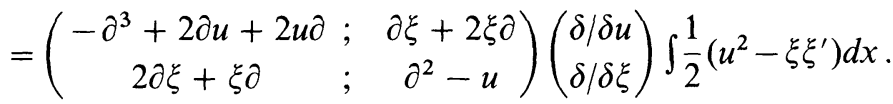


From this representation it is clear that $(1.1) /(1.7)$ reduce to the "second Hamiltonian formulation" of the $\mathrm{KdV}$ when passing to the bosonic sector, i.e. putting $\xi \equiv 0$. No other supersymmetric Hamiltonian formulation of (1.1) had been found having the property of reducing to the well known "first Hamiltonian formulation" of the $\mathrm{KdV}$ in the bosonic sector. This will be explained later on: we found that the proper supersymmetric "first Hamiltonian formulation" for (1.1)/(1.7) is an "inverse" Hamiltonian formulation using a suitable (pre-)symplectic 2-form instead of a Hamiltonian operator (Poisson tensor). This reduces to the "inverse first Hamiltonian formulation" of the $\mathrm{KdV}$ in the bosonic sector.

The case of the $N=2$ superconformal algebra also yields interesting integrable extensions of the $\mathrm{KdV}$. These extensions consist of two bosonic and two fermionic fields interacting among themselves. In [10] three such integrable extensions are given, one of them was found to be bi-Hamiltonian but it is not invariant with respect to supersymmetric transformations. We will be interested only in the two other cases, which are supersymmetric invariant. Both cases are contained in the following one-parameter family of super-Hamiltonian evolution equations:

$$
\begin{aligned}
\Phi_{t} & =-\Phi^{\prime \prime \prime}+3\left(\Phi D_{1} D_{2} \Phi\right)^{\prime}+\frac{1}{2}(\alpha-1)\left(D_{1} D_{2} \Phi^{2}\right)^{\prime}+3 \alpha \Phi^{2} \Phi^{\prime} \\
& =\left(D_{1} D_{2} \partial+2 \partial \Phi+2 \Phi \partial-D_{1} \Phi D_{1}-D_{2} \Phi D_{2}\right) \frac{\delta}{\delta \Phi} \int \frac{1}{2}\left(\Phi D_{1} D_{2} \Phi+\frac{\alpha}{3} \Phi^{3}\right) d X
\end{aligned}
$$

where $\alpha$ is an arbitrary constant. Here $\Phi=\Phi\left(x, \Theta_{1}, \Theta_{2}\right)$ is a commuting superfunction of space and two Grassmannian variables $\Theta_{1}$ and $\Theta_{2}$. Again the prime indicates differentiation with respect to $x$, the symbols $D_{1}$ and $D_{2}$ are the superderivatives $D_{i}=\Theta_{i} \partial+\partial_{\Theta}$. The operator

$$
P_{2}(\Phi):=D_{1} D_{2} \partial+2 \partial \Phi+2 \Phi \partial-D_{1} \Phi D_{1}-D_{2} \Phi D_{2}
$$

in (1.8) is the Hamiltonian operator stemming from the $N=2$ extension of the Virasoro algebra. The Hamiltonian function in (1.8) is obtained by integrating its commuting density $\frac{1}{2}\left(\Phi D_{1} D_{2} \Phi+\frac{\alpha}{3} \Phi^{3}\right)$ over $x$ and both Grassmannians, i.e. $d X=d x d \Theta_{1} d \Theta_{2}$ normalized by $\int \Theta_{i} d \Theta_{j}=\delta_{i j}$.

Although for arbitrary values of $\alpha$ a Miura transformation for (1.8) had been given in [10], only for the two cases $\alpha=-2$ and $\alpha=4$ Lax formulations had been found. They are given by

$$
\begin{aligned}
\alpha=-2: L & =\partial^{2}+D_{1} \Phi D_{2}-D_{2} \Phi D_{1}, \\
\alpha=4: L & =\partial^{2}-\left(D_{1} D_{2} \Phi\right)-\Phi^{2}+\left(D_{2} \Phi\right) D_{1}-\left(D_{1} \Phi\right) D_{2}-2 \Phi D_{1} D_{2} \\
& =-\left(D_{1} D_{2}+\Phi\right)^{2},
\end{aligned}
$$

i.e. for these special choices of $\alpha$ equation (1.8) is equivalent to $d / d t L=$ $\left[-4\left(L^{3 / 2}\right)_{+}, L\right]$.

Expanding the superfunction $\Phi$ into powers of the Grassmannians we may assume $\Phi\left(x, \Theta_{1}, \Theta_{2}\right)=w(x)+\Theta_{1} \xi_{2}(x)+\Theta_{2} \xi_{1}(x)+\Theta_{2} \Theta_{1} u(x)$ with two bosonic fields $u$ and $w$ and two fermionic fields $\xi_{1}$ and $\xi_{2}$. Corresponding to this chart we represent vector fields $K$ and co-vector fields $\gamma$ as

$$
\begin{gathered}
K(\Phi)=K_{w}+\Theta_{1} K_{\xi_{2}}+\Theta_{2} K_{\xi_{1}}+\Theta_{2} \Theta_{1} K_{u}, \\
\gamma(\Phi)=\gamma_{u}+\gamma_{\xi_{1}} \Theta_{1}-\gamma_{\xi_{2}} \Theta_{2}+\gamma_{w} \Theta_{2} \Theta_{1},
\end{gathered}
$$


such that the duality becomes the usual

$$
\langle K, \gamma\rangle=\int K \gamma d x d \Theta_{1} d \Theta_{2}=\int\left(K_{u} \gamma_{u}+K_{w} \gamma_{w}+K_{\xi_{1}} \gamma_{\xi_{1}}+K_{\xi_{2}} \gamma_{\xi_{2}}\right) d x
$$

Regarding $P_{2}(\Phi)$ as a linear map from the co-vector fields to the vector fields the Hamiltonian system (1.8) now can be decomposed into

$$
\frac{d}{d t}\left(\begin{array}{c}
u \\
\xi_{1} \\
\xi_{2} \\
w
\end{array}\right)=\left(\begin{array}{l}
\partial\left(-u^{\prime \prime}+3 u^{2}-3 \xi_{1} \xi_{1}^{\prime}-3 \xi_{2} \xi_{2}^{\prime}-(\alpha-1)\left(w^{\prime}\right)^{2}-(\alpha+2) w w^{\prime \prime}+3 \alpha u w^{2}+6 \alpha w \xi_{2} \xi_{1}\right) \\
\partial\left(-\xi_{1}^{\prime \prime}+3 u \xi_{1}+3 \alpha w^{2} \xi_{1}-(\alpha+2) w \xi_{2}^{\prime}-(\alpha-1) w^{\prime} \xi_{2}\right) \\
\partial\left(-\xi_{2}^{\prime \prime}+3 u \xi_{2}+3 \alpha w^{2} \xi_{2}+(\alpha+2) w \xi_{1}^{\prime}+(\alpha-1) w^{\prime} \xi_{1}\right) \\
\partial\left(-w^{\prime \prime}+\alpha w^{3}+(\alpha+2) u w+(\alpha-1) \xi_{2} \xi_{1}\right)
\end{array}\right)
$$

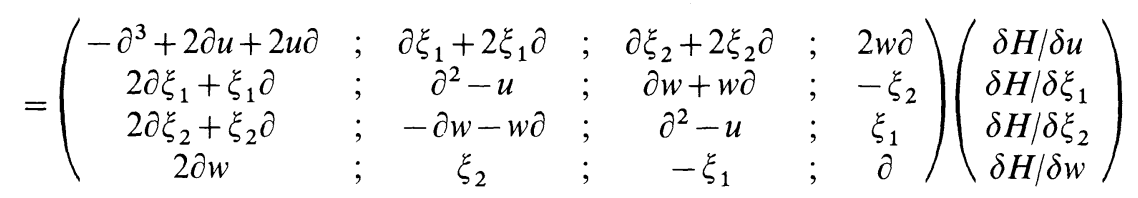

with the Hamiltonian function

$$
\begin{aligned}
H & =\frac{1}{2} \int\left(\Phi D_{1} D_{2} \Phi+\frac{\alpha}{3} \Phi^{3}\right) d X \\
& =\frac{1}{2} \int\left(u^{2}-w w^{\prime \prime}-\xi_{1} \xi_{1}^{\prime}-\xi_{2} \xi_{2}^{\prime}+\alpha u w^{2}-2 \alpha w \xi_{1} \xi_{2}\right) d x .
\end{aligned}
$$

From these representations it becomes obvious that we will recover the "second Hamiltonian formulation" of the $\mathrm{KdV}$ if we reduce $(1.8) /(1.13)$ by claiming $\xi_{1}=\xi_{2}=w \equiv 0$. Again, just as in the $N=1$ case the question arises, whether there is a further supersymmetric Hamiltonian formulation of $(1.8) /(1.13)$, for the integrable cases $\alpha=-2,4$ yielding the well known "first Hamiltonian formulation" of the $\mathrm{KdV}$ in the above reduction.

In [10] it was claimed that no such "first Hamiltonian formulation" for $(1.8) /(1.13), \alpha=-2,4$ exists. It turns out that this claim is based on rather restrictive assumptions on the explicit form of such a formulation, and we will show that this formulation does indeed exist.

In Sect. 2 we will give the missing "first Hamiltonian formulation" for all the integrable cases $N=1$ and $N=2, \alpha=-2,4$. In each case a fully supersymmetric hereditary recursion operator [11] arises giving direct access to the symmetries and conservation laws of these systems. Furthermore, starting from the conformal invariance of these equations, a hierarchy of master symmetries [12-14] can be obtained yielding a convenient recursive scheme to obtain the higher conservation laws. In Sect. 3 we will briefly indicate how the bi-Hamiltonian formulations can be derived in a systematic way from the Lax formulation. This also will show how further-more complicated-supersymmetric bi-Hamiltonian extensions of the KdV can be constructed in a straightforward way. This shall be demonstrated by deriving a further integrable extension of the $\mathrm{KdV}$ which yields the $N=1$ extension (1.1) by a simple restriction. 


\section{Results}

2.1. The Case $N=1$. For Eq. (1.1)/(1.7) we found the following "inverse" Hamiltonian formulation

$$
\begin{aligned}
\left(D \partial^{-1}-\partial^{-1} \Phi \partial^{-1}\right)\left(-\Phi^{\prime \prime}+3 \Phi D \Phi\right)^{\prime} & =-D \Phi^{\prime \prime}-2 \Phi \Phi^{\prime}+3(D \Phi)^{2} \\
& =\frac{\delta}{\delta \Phi} \int\left(\frac{1}{2} \Phi^{\prime} D \Phi^{\prime}+\Phi(D \Phi)^{2}\right) d X
\end{aligned}
$$

where the operator

$$
J(\Phi):=D \partial^{-1}-\partial^{-1} \Phi \partial^{-1}
$$

in (2.1) defines a closed (i.e. pre-symplectic) 2-form $\omega_{J}$

$$
\omega_{J}(\Phi)(a, b)=\int a J(\Phi) b d X=-\omega_{J}(\Phi)(b, a)
$$

over the space spanned by the anticommuting field $\Phi$. In (2.3) $a$ and $b$ are arbitrary even vector fields (i.e. with anticommuting values). For completeness we also state the matrix version of the operator $J$. Using the chart $\Phi=\xi+\Theta u$ introduced in the last section the operator $J$ (regarded as map from the vector fields to the co-vector fields) translates to the matrix operator

$$
J(u, \xi)=\left(\begin{array}{ccc}
\partial^{-1} & ; & -\partial^{-1} \xi \partial^{-1} \\
-\partial^{-1} \xi \partial^{-1} & ; & -1+\partial^{-1} u \partial^{-1}
\end{array}\right) .
$$

Inverse Hamiltonian formulations are familiar in the theory of bi-Hamiltonian systems [15-17]. The "closed" operator $J(2.2) /(2.4)$ is compatible [15] with the Hamiltonian operator $P_{2}$ in (1.2)/(1.7), i.e. the resulting recursion operator

$$
R(\Phi):=P_{2}(\Phi) J(\Phi)
$$

turns out to be hereditary. As in the usual bi-Hamiltonian scheme [11] we can construct a hierarchy $K_{n}:=R^{n-1} K_{1}$ of symmetry generators for (1.1)/(1.7) starting with any simple symmetry generator $K_{1}$ for this equation. Choosing $K_{1}$ to be space translation (which also leaves $R$ invariant) we thus have found an infinite sequence of commuting vector fields. The first three are given by

$$
\begin{aligned}
& K_{1}(\Phi)=\partial \Phi \\
& K_{2}(\Phi)=\partial\left(-\Phi^{\prime \prime}+3 \Phi D \Phi\right) \\
& K_{3}(\Phi)=\partial\left(\Phi^{\prime \prime \prime \prime}-5 \Phi^{\prime} D \Phi^{\prime}-5 \Phi D \Phi^{\prime \prime}-5(D \Phi) \Phi^{\prime \prime}+10 \Phi(D \Phi)^{2}\right)
\end{aligned}
$$

As in the usual bi-Hamiltonian scheme there exists a hierarchy of conserved functionals $H_{m}$ for the vector fields $K_{n}$, these turn out to be bi-Hamiltonian again:

$$
J K_{n}=\frac{\delta}{\delta \Phi} H_{n-1}, K_{n}=P_{2} \frac{\delta}{\delta \Phi} H_{n-2}, \quad n=1,2,3, \ldots
$$

The first of these Hamiltonian functions read

$$
\begin{aligned}
H_{-1}(\Phi) & =\int \frac{1}{2} \Phi d X \\
H_{0}(\Phi) & =\int \frac{1}{2} \Phi D \Phi d X \\
H_{1}(\Phi) & =\int\left(\frac{1}{2} \Phi^{\prime} D \Phi^{\prime}+\Phi(D \Phi)^{2}\right) d X \\
H_{2}(\Phi) & =\int\left(\frac{1}{2} \Phi^{\prime \prime} D \Phi^{\prime \prime}+5 \Phi\left(D \Phi^{\prime}\right)^{2}-3 \Phi \Phi^{\prime} \Phi^{\prime \prime}+\frac{5}{2} \Phi(D \Phi)^{3}\right) d X
\end{aligned}
$$


they coincide with the conserved quantities found in [9]. As all objects considered here are graded there is a conformal symmetry generated by the vector field

$$
\tau_{0}(\Phi):=x \Phi^{\prime}+\frac{1}{2} \Theta(D \Phi)+\frac{3}{2} \Phi \equiv\left(\begin{array}{l}
x u^{\prime}+2 u \\
x \xi^{\prime}+\frac{3}{2} \xi
\end{array}\right)=: \tau_{0}(u, \xi) .
$$

Using the recursion operator we can define a sequence of master symmetries by $\tau_{n}:=R^{n} \tau_{0}$, satisfying the "standard scheme" of bi-Hamiltonian systems admitting scaling invariance $[13,14]$ :

$$
\begin{aligned}
& {\left[\tau_{n}, K_{m}\right]=(2 m-1) K_{n+m},\left[\tau_{n}, \tau_{m}\right]=2(m-n) \tau_{n+m},} \\
& \int \tau_{n} \frac{\delta H_{m}}{\delta \Phi} d X=(2(m+n)+3) H_{n+m} .
\end{aligned}
$$

The last relation may be considered as a convenient scheme for a direct recursive construction of the conserved quantities using $\tau_{1}$, say.

2.2. The Case $N=2, \alpha=-2$. For Eq. (1.8)/(1.13) with $\alpha=-2$ we found the following "inverse" Hamiltonian formulation

$$
\begin{gathered}
J(\Phi)\left(-\Phi^{\prime \prime}+3 \Phi D_{1} D_{2} \Phi-\frac{3}{2} D_{1} D_{2} \Phi^{2}-2 \Phi^{3}\right)^{\prime} \\
=\frac{\delta}{\delta \Phi} \int\left(-\frac{1}{2}\left(D_{1} \Phi^{\prime}\right)\left(D_{2} \Phi^{\prime}\right)-2 \Phi\left(\Phi^{\prime}\right)^{2}+\Phi\left(D_{1} D_{2} \Phi\right)^{2}-\Phi^{3}\left(D_{1} D_{2} \Phi\right)+\frac{3}{5} \Phi^{5}\right) d X
\end{gathered}
$$

where the operator

$$
J(\Phi):=\left(D_{1} D_{2} \partial^{-1}-\partial^{-1} D_{1} \Phi D_{1} \partial^{-1}-\partial^{-1} D_{2} \Phi D_{2} \partial^{-1}\right)
$$

in (2.11) defines via (2.3) a (pre-)symplectic 2 -form $\omega_{J}$ over the space spanned by the commuting field $\Phi$. Again the vector fields $a$ and $b$ in (2.3) have to be even, i.e. now with commuting values. Using the chart $\Phi=w+\Theta_{1} \xi_{2}+\Theta_{2} \xi_{1}+\Theta_{2} \Theta_{1} u$ introduced in the last section the operator $J$ (regarded as map from the vector fields to the co-vector fields) translates to the matrix operator

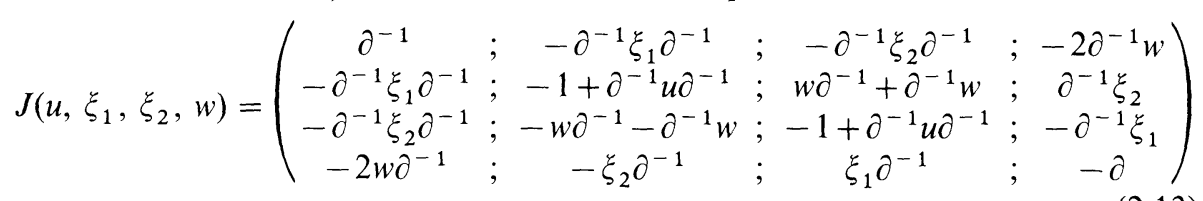

Again, the resulting recursion operator $R(\Phi):=P_{2}(\Phi) J(\Phi)$ turns out to be hereditary. As before we can construct the hierarchy $K_{n}:=R^{n-1} K_{1}$ of commuting symmetry generators for $(1.8) /(1.13)$ starting with the generator of space translation $K_{1}(\Phi):=\Phi^{\prime}$. The first of these symmetries read

$$
\begin{aligned}
K_{1}(\Phi)= & \partial \Phi \\
K_{2}(\Phi)= & \partial\left(-\Phi^{\prime \prime}+3\left(\Phi D_{1} D_{2} \Phi\right)-\frac{3}{2}\left(D_{1} D_{2} \Phi^{2}\right)-2 \Phi^{3}\right) \\
K_{3}(\Phi)= & \partial\left(\Phi^{\prime \prime \prime \prime}+5\left(D_{1} \Phi\right)\left(D_{2} \Phi^{\prime \prime}\right)+5\left(D_{1} \Phi^{\prime \prime}\right)\left(D_{2} \Phi\right)+5\left(D_{1} \Phi^{\prime}\right)\left(D_{2} \Phi^{\prime}\right)\right. \\
& -10\left(D_{1} D_{2} \Phi\right)\left(D_{1} \Phi\right)\left(D_{2} \Phi\right)+10 \Phi^{2} \Phi^{\prime \prime}+10 \Phi\left(\Phi^{\prime}\right)^{2} \\
& \left.+30 \Phi^{2}\left(D_{1} \Phi\right)\left(D_{2} \Phi\right)+6 \Phi^{5}\right) .
\end{aligned}
$$

As before we can grant the existence of a hierarchy of conserved functionals $H_{m}$ for 
the vector fields $K_{n}$; these turn out to be bi-Hamiltonian again:

$$
J K_{n}=\frac{\delta H_{n-1}}{\delta \Phi}, K_{n}=P_{2} \frac{\delta H_{n-2}}{\delta \Phi}, \quad n=1,2,3, \ldots
$$

The first of these Hamiltonian functions read

$$
\begin{aligned}
H_{-1}(\Phi)= & \int \frac{1}{2} \Phi d X \\
H_{0}(\Phi)= & \int\left(\frac{1}{2} \Phi D_{1} D_{2} \Phi-\frac{1}{2} \Phi^{3}\right) d X \\
H_{1}(\Phi)= & \int\left(-\frac{1}{2}\left(D_{1} \Phi^{\prime}\right)\left(D_{2} \Phi^{\prime}\right)-2 \Phi\left(\Phi^{\prime}\right)^{2}+\Phi\left(D_{1} D_{2} \Phi\right)^{2}\right. \\
& \left.-\Phi^{3}\left(D_{1} D_{2} \Phi\right)+\frac{3}{5} \Phi^{5}\right) d X
\end{aligned}
$$

As all objects considered here are graded there again is a conformal symmetry generated by the vector field

$$
\tau_{0}(\Phi):=x \Phi^{\prime}+\frac{1}{2} \Theta_{1}\left(D_{1} \Phi\right)+\frac{1}{2} \Theta_{2}\left(D_{2} \Phi\right)+\Phi \equiv\left(\begin{array}{l}
x u^{\prime}+2 u \\
x \xi_{1}^{\prime}+\frac{3}{2} \xi_{1} \\
x \xi_{2}^{\prime}+\frac{3}{2} \xi_{2} \\
x w^{\prime}+w
\end{array}\right)=: \tau_{0}\left(u, \xi_{1}, \xi_{2}, w\right) \text {. }
$$

Again just as in the $N=1$ case the master symmetries generated from the conformal symmetry by $\tau_{n}:=R^{n} \tau_{0}$ satisfy the scheme (2.10) (with the same coefficients). Hence all the higher conservation laws can be constructed directly using this recursive formula with $\tau_{1}$, say.

2.3. The Case $N=2, \alpha=4$. For Eq. (1.8)/(1.13) with $\alpha=4$ the "first" Hamiltonian formulation is given by

$$
\begin{aligned}
\Phi_{t} & =\partial\left(-\Phi^{\prime \prime}+3 \Phi D_{1} D_{2} \Phi+\frac{3}{2} D_{1} D_{2} \Phi^{2}+4 \Phi^{3}\right) \\
& =\partial \frac{\delta}{\delta \Phi} \int\left(\frac{1}{2}\left(\Phi^{\prime}\right)^{2}+\frac{3}{2} \Phi^{2} D_{1} D_{2} \Phi+\Phi^{4}\right) d X
\end{aligned}
$$

where the operator

$$
P_{1}(\Phi):=\partial
$$

constitutes a Hamiltonian operator for this system. This Hamiltonian formulation had been observed by B. Kupershmidt [18]. The operator (2.19) decomposes into

$$
P_{1}\left(u, \xi_{1}, \xi_{2}, w\right)=\left(\begin{array}{cccc}
0 & 0 & 0 & \partial \\
0 & 0 & \partial & 0 \\
0 & -\partial & 0 & 0 \\
\partial & 0 & 0 & 0
\end{array}\right)
$$

when introducing $\Phi=w+\Theta_{1} \xi_{2}+\Theta_{2} \xi_{1}+\Theta_{2} \Theta_{1} u$. Clearly $P_{1}$ and $P_{2}$ given by (1.9) are compatible, as the deformation $\Phi \rightarrow \Phi+\varepsilon 1$ maps $P_{2}$ into the Hamiltonian operator $P_{2}+\varepsilon 2 P_{1}$. Hence the resulting recursion operator $R(\Phi):=P_{2}(\Phi) P_{1}^{-1}(\Phi)=D_{1} D_{2}+2 \partial \Phi \partial^{-1}-\left(D_{1} \Phi\right) D_{1} \partial^{-1}-\left(D_{2} \Phi\right) D_{2} \partial^{-1}$ is hereditary. As before we can construct the hierarchy $K_{n}:=R^{n-1} K_{1}$ of commuting 
symmetry generators for (2.18) starting with the generator of space translation $K_{1}(\Phi):=\Phi^{\prime}$. The first of these symmetries read

$$
\begin{aligned}
& K_{1}(\Phi)=\partial \Phi \\
& K_{2}(\Phi)=\partial\left(D_{1} D_{2} \Phi+2 \Phi^{2}\right) \\
& K_{3}(\Phi)=\partial\left(-\Phi^{\prime \prime}+3 \Phi D_{1} D_{2} \Phi+\frac{3}{2} D_{1} D_{2} \Phi^{2}+4 \Phi^{3}\right)
\end{aligned}
$$

These vector fields are again bi-Hamiltonian

$$
J K_{n}=\frac{\delta H_{n-1}}{\delta \Phi}, K_{n}=P_{2} \frac{\delta H_{n-2}}{\delta \Phi}, \quad n=1,2,3, \ldots,
$$

where the first of the Hamiltonian functions read

$$
\begin{aligned}
H_{-1}(\Phi)= & \int \frac{1}{2} \Phi d X=\int \frac{1}{2} u d x, \\
H_{0}(\Phi)= & \int \frac{1}{2} \Phi^{2} d X=\int\left(u w-\xi_{1} \xi_{2}\right) d x, \\
H_{1}(\Phi)= & \int\left(\frac{1}{2} \Phi D_{1} D_{2} \Phi+\frac{2}{3} \Phi^{3}\right) d X \\
= & \int\left(\frac{1}{2} u^{2}-\frac{1}{2} w w^{\prime \prime}-\frac{1}{2} \xi_{1} \xi_{1}^{\prime}-\frac{1}{2} \xi_{2} \xi_{2}^{\prime}+2 u w^{2}-4 w \xi_{1} \xi_{2}\right) d x, \\
H_{2}(\Phi)= & \int\left(\frac{1}{2}\left(\Phi^{\prime}\right)^{2}+\frac{3}{2} \Phi^{2} D_{1} D_{2} \Phi+\Phi^{4}\right) d X \\
= & \int\left(u^{\prime} w^{\prime}-\xi_{1}^{\prime} \xi_{2}^{\prime}+3 w\left(w^{\prime}\right)^{2}-3 w \xi_{1} \xi_{1}^{\prime}-3 w \xi_{2} \xi_{2}^{\prime}\right. \\
& \left.+3 w u^{2}-3 u \xi_{1} \xi_{2}+4 u w^{3}-12 w^{2} \xi_{1} \xi_{2}\right) d x .
\end{aligned}
$$

A conformal symmetry for all objects is again generated by the vector field $\tau_{0}$ defined in (2.17). Following $[13,14]$ we now find a recursive scheme for the master symmetries $\tau_{n}:=R^{n} \tau_{0}$ given by

$$
\begin{aligned}
{\left[\tau_{n}, K_{m}\right] } & =m K_{n+m},\left[\tau_{n}, \tau_{m}\right]=(m-n) \tau_{n+m}, \\
\int \tau_{n} \frac{\delta H_{m}}{\delta \Phi} d X & =(m+n+2) H_{n+m} .
\end{aligned}
$$

We remark that in this case there is an additional master symmetry

$$
\tau_{-1}(\Phi):=\frac{1}{2} \equiv\left(\begin{array}{c}
0 \\
0 \\
0 \\
1 / 2
\end{array}\right)=: \tau_{-1}\left(u, \xi_{1}, \xi_{2}, w\right)
$$

fitting into the algebraic scheme (2.25). The flow generated by $\tau_{-1}$ corresponds to the Galilean boost admitted by the $\mathrm{KdV}$; it provides the deformation between the Hamiltonian operators $P_{2}$ and $P_{1}$.

Considering the explicit forms of the first Hamiltonian operator $P_{1}$ and the Hamiltonian functions $H_{n}$ it now becomes clear why these structures had not been found before in [10]. The Hamiltonian formulations considered in [10] were assumed to admit o(2)-invariance in the fermionic sector; all objects should be graded and the reduction $w=\xi_{2}=0$ should yield the Hamiltonian formulation of the $N=1$ case. Although this last assumption seems reasonable (note that (1.13) reduces to (1.7) when putting $w=\xi_{2}=0$ ) it now turns out that this last hypothesis had been too restrictive. For all even indices the Hamiltonian functions $H_{n}$ defined above vanish identically in this reduction; the Hamiltonian operator $P_{1}$ cannot be reduced to the subspace of vanishing $w$ and $\xi_{2}$. Also the recursion operator (2.21) 
is not reducible, but its square is (reducing to the recursion operator defined with (2.5) by (1.2) and (2.2). From this we can conclude that in the hierarchy of symmetries $K_{n}$ only each second member (those with odd indices) can be reduced to the $N=1$ case, whereas the symmetries with even indices are additional structures peculiar to the case $N=2, \alpha=4$. Indeed, observe that now Eq. (2.18) given by the vector field $K_{3}$ is not the first nonlinear equation in the hierarchy (2.22), but there is a "simpler" nonlinear symmetry $K_{2}$ with components

$$
K_{2}\left(u, \xi_{1}, \xi_{2}, w\right)=\left(\begin{array}{l}
\partial\left(-w^{\prime \prime}+4 u w-4 \xi_{1} \xi_{2}\right) \\
\partial\left(-\xi_{2}^{\prime}+4 w \xi_{1}\right) \\
\partial\left(\xi_{1}^{\prime}+4 w \xi_{2}\right) \\
\partial\left(u+2 w^{2}\right)
\end{array}\right)
$$

with no corresponding symmetry in the cases $N=1$ and $N=2, \alpha=-2$. Also among the master symmetries $\tau_{n}$ only each second (with even index) is reducible to the corresponding master symmetries of the $N=1$ case. The first "additional" master symmetry reads

$$
\tau_{1}=x K_{2}+\left(\begin{array}{c}
8 u w-3 w^{\prime \prime}-9 \xi_{1} \xi_{2}-\frac{1}{2} \xi_{1}^{\prime} \partial^{-1} \xi_{2}-\frac{1}{2} \xi_{2}^{\prime} \partial^{-1} \xi_{1} \\
-\frac{5}{2} \xi_{2}^{\prime}+6 w \xi_{1}+\frac{1}{2} w^{\prime} \partial^{-1} \xi_{1}+\frac{1}{2} u^{\prime} \partial^{-1} \xi_{2}-\xi_{2} \partial^{-1} u \\
\frac{5}{2} \xi_{1}^{\prime}+6 w \xi_{2}+\frac{1}{2} w^{\prime} \partial^{-1} \xi_{2}-\frac{1}{2} u^{\prime} \partial^{-1} \xi_{1}+\xi_{1} \partial^{-1} u \\
2 u+2 w^{2}-\frac{1}{2} \xi_{1} \partial^{-1} \xi_{1}-\frac{1}{2} \xi_{2} \partial^{-1} \xi_{2}
\end{array}\right)
$$

Note that all higher symmetries and Hamiltonian functions can be obtained from this simple vector field using the recursive scheme (2.25).

There is a simple "explanation" why in the $N=2, \alpha=4$ case there are additional invariants with no analogues in the other cases. Note that in terms of the Lax operators (1.4), (1.1) the integrable equation (1.1) and the two integrable cases of (1.8) are represented by $d / d t L=\left[-4\left(L^{3 / 2}\right)_{+}, L\right]$. The dynamical systems

$$
\frac{d}{d t_{n}} \Phi=K_{n}(\Phi)=R(\Phi)^{n-1} \Phi^{\prime}
$$

associated to the symmetries of such equations usually can be obtained by Lax equations to the form

$$
\frac{d}{d t_{n}} L=\operatorname{const}(n)\left[\left(L^{k(n)}\right)_{+}, L\right]
$$

with suitable fractional powers $k(n)$ of $L$ (see e.g. [19, 20]) and suitable coefficients const $(n)$. The subscript + again indicates the pure differential part of the operators. Here the fractional powers of $L$ can obtained from the square roots of the Lax operators defined by the following expansion into (inverse) powers of the differential operator

$$
L^{1 / 2}=\partial+\sum_{k=1}^{\infty}\left(b_{k}+\beta_{k} D_{1}+\gamma_{k} D_{2}+a_{k} D_{1} D_{2}\right) \partial^{-k} .
$$

Having fixed the "highest" term (i.e. the coefficient of the highest power of $\partial$ ) all the coefficients in the above expansion are determined uniquely by a recursive scheme obtained from the condition $L^{1 / 2} L^{1 / 2}=L$. We note that in the presence of 
superderivatives $D_{i}$ satisfying $D_{i}^{2}=\partial$ we need not necessarily assume the square root of an operator $L=\partial^{2}+\cdots$ to be given by $L^{1 / 2}=\partial+\cdots$. Indeed, as observed in [10] for the case $\alpha=4$ the Lax operator can be factorized (see (1.10.ii)) and there is an alternative "square root" (of $-L$ ):

$$
\hat{L}(\Phi):=D_{1} D_{2}+\Phi=(-L)^{1 / 2} .
$$

Now also Lax equations of the type

$$
\frac{d}{d t_{n}} L=\operatorname{const}(n)\left[\left(L^{k(n)} \hat{L}\right)_{+}, L\right]
$$

can be considered in addition to (2.30). Note that $\hat{L}$ commutes with the operator $L^{1 / 2}$ : introducing $\Delta:=\left[\hat{L}, L^{1 / 2}\right]$ it is easy to deduce from $\hat{L}^{2}=-\left(L^{1 / 2}\right)^{2}$ that $\Delta$ anticommutes with $L^{1 / 2}$, i.e. $L^{1 / 2} \Delta+\Delta L^{1 / 2}=0$. Considering the terms with the highest power of $\partial$ in $\Delta$ and using the explicit form of the highest terms in (2.31) one immediately concludes that the highest term of $\Delta$ has to vanish. Hence $\Delta$ has no highest term and must vanish identically. As $\hat{L}$ commutes with $L^{1 / 2}$ the equations (2.33) seem to represent the only candidates for additional interesting Lax equations. It turns out that the symmetries (2.22) with odd indices (reducible to the $N=1$ case) correspond to the Lax equations (2.30), whereas the additional (non-reducible) symmetries (2.22) correspond to (2.33). E.g., for the first symmetries it is simple to verify that

$$
\begin{aligned}
{\left[\left(L^{1 / 2}\right)_{+}, \hat{L}\right] } & =\Phi^{\prime} \\
{\left[\left(L^{1 / 2} \hat{L}\right)_{+}, \hat{L}\right] } & =\frac{1}{2}\left(D_{1} D_{2} \Phi+2 \Phi^{2}\right)^{\prime} \\
{\left[\left(L^{3 / 2}\right)_{+}, \hat{L}\right] } & =-\frac{1}{4}\left(-\Phi^{\prime \prime}+3 \Phi D_{1} D_{2} \Phi+\frac{3}{2} D_{1} D_{2} \Phi^{2}+4 \Phi^{3}\right)^{\prime},
\end{aligned}
$$

where for convenience we now have chosen $\hat{L}$ as the Lax operator instead of $L$. Indeed, from the derivation of the two Hamiltonian formulations from the Laxoperators (briefly indicated in the next section) it is easy to conclude that the Lax equations for (2.29) are given by

$$
\frac{d}{d t_{n}} \Phi=K_{n}(\Phi) \Longleftrightarrow \frac{d}{d t_{n}} \hat{L}=\left[2^{n-1}\left(L^{1 / 2} \hat{L}^{n-1}\right)_{+}, \hat{L}\right],
$$

such that from $\hat{L}^{2}=-L$ we find for the odd and even cases of $n$ :

$$
\begin{aligned}
& \frac{d}{d t_{2 k-1}} \hat{L}=\left[(-4)^{k-1}\left(L^{k-1 / 2}\right)_{+}, \hat{L}\right], \quad k=1,2, \ldots, \\
& \frac{d}{d t_{2 k}} \hat{L}=\left[2(-4)^{k-1}\left(L^{k-1 / 2} \hat{L}\right)_{+}, \hat{L}\right], \quad k=1,2, \ldots
\end{aligned}
$$

The Lax formulations of (2.29) in terms of the Lax operator $L$ follow from $(2.35) /(2.36)$ trivially.

Furthermore we notice that from the Lax operator $\hat{L}$ additional conserved quantities for (2.35) are given by the residues of the pseudo-differential operators $L^{k+1 / 2} \hat{L}$. These seem to correspond to the additional Hamiltonian functions $H_{2 k}$ which reduce to 0 when passing to the $N=1$ case.

We finally remark that all structures found for the case $N=2, \alpha=4$ admit a reduction to the bosonic sector, i.e. every object considered here survives when 
putting $\xi_{1}=\xi_{2}=0$. The remarkable fact about this reduction is that in contrast to most examples of integrable hierarchies with conformal invariance the first non-trivial master symmetry $\tau_{1}$ given by (2.28) reduces to a local vector field containing no integrational terms. Now it is interesting to consider a third Hamiltonian operator $P_{3}:=R P_{2}$, which can also be obtained by the Lie derivative of $P_{2}$ into the direction of $\tau_{1}[13,14]$. As now both $P_{2}$ and $\tau_{1}$ are local objects, $P_{3}$ is again local, i.e. a purely differential operator. No further local Hamiltonian operator can be derived that way, as the Lie derivative of $P_{3}$ into the direction of $\tau_{1}$ vanishes. To sum up: the bosonic parts of all equations (2.29) admit three local Hamiltonian formulations with the differential operators $P_{1}, P_{2}$ and $P_{3}$.

\section{The Method}

3.1. General Background. The results of the last section, i.e. essentially the revelation of the proper bi-Hamiltonian structure, had not been found accidentally but were derived in a systematic way from the Lax operators. Indeed, it turns out that there is a natural way of associating several Hamiltonian structures to a given Lax operator yielding multi-Hamiltonian formulations for the isospectral flows connected to the scattering problem given by that Lax operator. Following [20-23] we consider an abstract integrable system to be defined on some associative algebra $g=g_{+} \oplus g_{-}$, that can be decomposed into 2 subalgebras $g_{ \pm}$. Let us assume that there exists a "traceform" $\operatorname{tr}: g \rightarrow \mathbb{R}$, such that $\langle a, b\rangle:=\operatorname{tr}(a b)$ defines a nondegenerate symmetric metric on $g$. If $\left\langle g_{+}, g_{+}\right\rangle=0=\left\langle g_{-}, g_{-}\right\rangle$(i.e. $g_{\mp}$ can be regarded as the dual space $g_{ \pm}^{*}$ relative to this trace pairing) then there exist two natural compatible Hamiltonian operators $P_{1}$ and $P_{2}$ on $g_{+}$given by the linear maps

$$
\begin{aligned}
& P_{1}(L): \nabla H \in g_{-} \rightarrow\left(L(\nabla H)_{-}\right)_{+}-\left((\nabla H)_{-} L\right)_{+} \in g_{+}, \\
& P_{2}(L): \nabla H \in g_{-} \rightarrow L\left((\nabla H)_{-} L\right)_{+}-\left(L(\nabla H)_{-}\right)_{+} L \in g_{+},
\end{aligned}
$$

where the subscripts \pm are to denote the projections onto the subalgebras $g_{ \pm}$. The first bracket in (3.1) is the Lie Poisson structure associated to the (Lie) algebra of Lax operators, leading to a "first" Hamiltonian formulation of the iso-spectral equations in the celebrated Adler-Kostant-Symes scheme [20]. Indeed, we will find that this abstract bracket will reduce to the "first" Hamiltonian formulation of the examples investigated here. The algebraic foundation of the bracket engendered by $P_{2}$ in (3.1) was revealed in [21], this abstract bracket will be shown to lead to the "second" Hamiltonian structure for our examples.

The essential observation is that invarient functions on $g$ (i.e. functions $C: g \rightarrow \mathbb{R}$ with gradients $\nabla C$ satisfying $\nabla C(L) L=L \nabla C(L), L \in g)$ are in involution with respect to both Poisson brackets engendered by these tensors. For the invariant functions $C_{k}:=\operatorname{tr}\left(L^{k}\right) / k$ given by the traceform one has $\nabla C_{k}=C_{k-1}$, such that the "integrable" Hamiltonian equations associated to this hierarchy of function in involution obtain the simple (bi-Hamiltonian) Lax-form

$$
\frac{d}{d t} L=\left[L,\left(L^{k}\right)_{-}\right] \equiv\left[\left(L^{k}\right)_{+}, L\right]=P_{1} \nabla C_{k+1}=\frac{1}{2} P_{2} \nabla C_{k} .
$$

For a more general discussion of the multi-Hamiltonian background we refer to 
[21-23] and the references therein. We claim that all the integrable hierarchies discussed here are just different realizations of the abstract bi-Hamiltonian hierarchy (3.2) by choosing the proper algebra $g$ for the corresponding Laxoperators. Hence the derivation of the Hamiltonian operators for these examples just boils down to evaluation of the abstract maps (3.1) after having embedded the Lax-operators (1.4), (1.10) into a suitable algebra of operators bearing a suitable traceform.

For the integrable extensions of the $\mathrm{KdV}$ discussed in the last sections the known Lax operators may be regarded as elements of the algebra of super pseudo-differential operators

for the case $N=1$ and

$$
g:=\left\{\sum_{k<\infty}\left(a_{k}+\Phi_{k} D\right) \partial^{k}\right\}
$$

$$
g:=\left\{\sum_{k<\infty}\left(b_{k}+\beta_{k} D_{1}+\gamma_{k} D_{2}+a_{k} D_{1} D_{2}\right) \partial^{k}\right\}
$$

for the case $N=2$. Here $a_{k}$ and $b_{k}$ are multiplication operators with commuting super functions of space and Grassmannian variables, whereas $\Phi_{k}, \gamma_{k}, \beta_{k}$ represent anticommuting super functions. The $D_{i}$ 's are the super derivatives arising from the Grassmannians. The action of the powers $\partial^{k}$ of the differential operator is to be understood by Leibniz' rule, i.e.

$$
\partial^{k} A=\sum_{j=0}^{\infty}\left(\begin{array}{c}
k \\
j
\end{array}\right) A^{(j)} \partial^{k-j},
$$

where $A, A^{(j)}$ are the multiplication operators with the function $A$ and its $x$-derivatives, respectively.

In both cases $N=1$ and $N=2$ the algebra $g$ can be decomposed into 2 subalgebras $g_{+}$and $g_{-}$given by the "purely differential parts" and the "purely integrational parts"

$$
\begin{array}{ll}
N=1: & g_{+}:=\left\{\Sigma_{0 \leq k<\infty}\left(a_{k}+\Phi_{k} D\right) \partial^{k}\right\}, \\
& g_{-}:=\left\{\Sigma_{k<0}\left(a_{k}+\Phi_{k} D\right) \partial^{k}\right\}, \\
N=2: & g_{+}:=\left\{\Sigma_{0 \leq k<\infty}\left(b_{k}+\beta_{k} D_{1}+\gamma_{k} D_{2}+a_{k} D_{1} D_{2}\right) \partial^{k}\right\}, \\
& g_{-}:=\left\{\Sigma_{k<0}\left(b_{k}+\beta_{k} D_{1}+\gamma_{k} D_{2}+a_{k} D_{1} D_{2}\right) \partial^{k}\right\},
\end{array}
$$

of the operators. We remark that for $N=2$ the subspace $g$ - does not define a proper subalgebra $g$ (with respect to multiplication of the operators) but rather a Lie subalgebra (with respect to the natural commutator). This suffices for the general results of [21] -i.e. the construction of the 2 compatible abstract brackets (3.1) - to hold for this example, too. These algebras are endowed with nondegenerate symmetric "trace-forms" given by the residues

$$
\operatorname{tr}(L):=\operatorname{Res}\left(\sum_{k<\infty}\left(a_{k}+\Phi_{k} D\right) \partial^{k}\right)=\int \Phi_{-1} d X
$$

for $N=1$ (with $d X=d x d \Theta)$ and

$$
\operatorname{tr}(L):=\operatorname{Res}\left(\sum_{k<\infty}\left(b_{k}+\beta_{k} D_{1}+\gamma_{k} D_{2}+a_{k} D_{1} D_{2}\right) \partial^{k}\right)=\int a_{-1} d X
$$

for $N=2$ (with $d X=d x d \Theta_{1} d \Theta_{2}$ ), respectively. 
As the Lax operators for the equations discussed before are known, one merely has to evaluate the abstract Poisson structures (3.1) at those points in $g$ given by these Lax operators. In a straightforward way this leads to the results presented before. The fact that in some cases these abstract Poisson structures need to be converted to an "inverse" Poisson structure, i.e. a symplectic 2 -form, turns up in a natural way from the reduction properties of these tensors. The decisive steps in the calculations for the various cases shall now be presented.

3.2. The Case $N=1$. The elements $L=\sum_{k \geqq 0}\left(a_{k}+\Phi_{k} D\right) \partial^{k}$ in $g_{+}$are to be para-
metrized by superfunctions

$$
a_{k}(x, \Theta)=b_{k}(x)+\Theta \zeta_{k}(x), \Phi_{k}(x, \Theta)=\xi_{k}(x)+\Theta u_{k}(x),
$$

with commuting fields $b_{k}, u_{k}$ and anticommuting field $\zeta_{k}$ and $\xi_{k}$. Given a Hamiltonian function $H(L)=H\left(\ldots, a_{k}, \Phi_{k}, \ldots\right)=H\left(\ldots, b_{k}, \zeta_{k}, \xi_{k}, u_{k}, \ldots\right)$ a convenient parametrization of the dual space $g_{-}$is given by representing the gradient $\nabla H$ as the operator

$$
\nabla H=\sum_{k \geqq 0} \partial^{-k-1}\left(-D \frac{\delta H}{\delta a_{k}}+\frac{\delta H}{\partial \Phi_{k}}\right),
$$

where

$$
\frac{\delta H}{\delta a_{k}}:=\frac{\delta H}{\delta \zeta_{k}}+\frac{\delta H}{\delta b_{k}} \Theta, \quad \frac{\delta H}{\delta \Phi_{k}}:=\frac{\delta H}{\delta u_{k}}+\frac{\delta H}{\delta \xi_{k}} \Theta,
$$

with commuting $\delta H / \delta \Phi_{k}$ and anticommuting $\delta H / \delta a_{k}$. Now the trace duality assumes the usual euclidean form

$$
\begin{aligned}
\langle\dot{L}, \nabla H\rangle=\operatorname{tr}(\dot{L} \nabla H) & =\sum_{k \geqq 0} \int\left(\dot{a}_{k} \frac{\delta H}{\delta a_{k}}+\dot{\Phi}_{k} \frac{\delta H}{\delta \Phi_{k}}\right) d X \\
& =\sum_{k \geqq 0} \int\left(\dot{b}_{k} \frac{\delta H}{\delta b_{k}}+\dot{\zeta}_{k} \frac{\delta H}{\delta \zeta_{k}}+\dot{\xi}_{k} \frac{\delta H}{\delta \xi_{k}}+\dot{u}_{k} \frac{\delta H}{\partial u_{k}}\right) d x .
\end{aligned}
$$

The functional derivatives $\delta / \delta a_{k}, \delta / \delta \Phi_{k}, \ldots, \delta / \delta \xi_{k}$ are defined by directional derivatives as in (1.3).

Now, trying to evaluate the first Poisson structure $P_{1}$ in (3.1) at the point $L=\partial^{2}-\Phi D \in g_{+}$one immediately encounters a technical difficulty: it turns out that the affine subspace $\left\{\partial^{2}-\Phi D\right\}$ of Lax operators (1.4) is not a Poisson submanifold of $P_{1}$, i.e. the corresponding Poisson bracket cannot be properly restricted to the points (1.4). Therefore we first embed these operators into the larger affine subspace of operators of the form

$$
L=\partial^{2}+a-\Phi D \text {. }
$$

The affine subspace spanned by the 2 superfunctions $a$ and $\Phi$ defines a proper Poisson submanifold. According to the dual parametrization (3.10) we put

$$
\nabla H=\partial^{-1}\left(-D \frac{\delta H}{\delta a}-\frac{\delta H}{\delta \Phi}\right)
$$

and insert this into (3.1). The Hamiltonian equation $\dot{L}=P_{1} \nabla H$ thus translates to

$$
\dot{L}=\dot{a}-\dot{\Phi} D=-2\left(\frac{\delta H}{\delta \Phi}\right)^{\prime}-2\left(D \frac{\delta H}{\delta a}\right)^{\prime}+2 \Phi \frac{\delta H}{\delta a}+2\left(\frac{\delta H}{\delta a}\right)^{\prime} D,
$$


such that the Hamiltonian equation associated to the abstract operator $P_{1}$ obtains the form

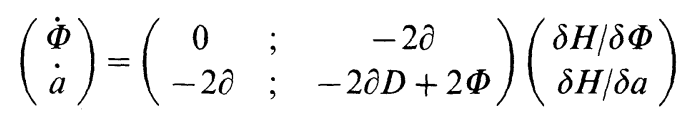

in the chart given by the coordinates $a$ and $\Phi$. This operator, providing a Hamiltonian formulation for the isospectral hierarchy of (3.13), had already been found in [24]. Now it is clearly seen that this Hamiltonian matrix cannot be properly restricted to the operators $L=\partial^{2}-\Phi D$. In order to pass from (3.13) to (1.4) we have to invoke Dirac reduction of the Poisson structure $P_{1}$ when imposing the constraint $a=0$. A discussion of such a reduction in suitable notation may be found in $[22,23]$, the procedure can be summarized as follows: Given a Poisson tensor

$$
P(u, v)=\left(\begin{array}{ll}
P_{u u}(u, v) & P_{u v}(u, v) \\
P_{v u}(u, v) & P_{v v}(u, v)
\end{array}\right)
$$

on the space spanned by the coordinates $u$ and $v$, then its Dirac reduction to the subspace spanned by $u$ (i.e. imposing the constraint $v=0$ ) is given by the reduced Hamiltonians operator

$$
P^{\text {(red) }}(u):=P_{u u}(u, 0)-P_{u v}(u, 0)\left(P_{v v}(u, 0)\right)^{-1} P_{v u}(u, 0),
$$

provided the above inverse of the matrix element $P_{v v}$ exists. Formally inverting the corresponding element in (3.16) we thus find a formal reduction

$$
P_{1}^{\text {(red) }}(\Phi)=2 \partial(\partial D-\Phi)^{-1} \partial
$$

providing a Hamiltonian operator for the hierarchy of integrable equations associated to the Lax operator (1.4). In order to avoid the formal inverse in (3.19) we consider the symplectic operator $J:=2\left(P_{1}^{(\text {red })}\right)^{-1}$ leading to (2.2).

Also the second Hamiltonian operator (1.2) associated to the $N=1$ superconformal algebra can be derived in the same way. The calculations now become much more troublesome, as the abstract map $P_{2}$ in (3.1) can neither be restricted to the operator subspace (1.4) spanned by $\Phi$ nor to the space (3.13) spanned by $a$ and $\Phi$. We thus first evaluate $P_{2}$ in (3.1) at the point (3.13), which should be regarded as the more general operator

$$
L=\partial^{2}+(b-\Psi D) \partial+a-\Phi D
$$

evaluated with $b=0$ and $\Psi=0$. According to the dual parametrization (3.10) we now have to put

$$
\nabla H=\partial^{-1}\left(-D \frac{\delta H}{\delta a}-\frac{\delta H}{\delta \Phi}\right)+\partial^{-2}\left(-D \frac{\delta H}{\delta b}-\frac{\delta H}{\delta \Psi}\right) .
$$

After some lengthy but straightforward calculation the Hamiltonian equation $\dot{L}=\dot{b} \partial-\dot{\Psi} D \partial+\dot{a}-\dot{\Phi} D=P_{2} \nabla H$ evaluated with $b=0, \Psi=0$ reads

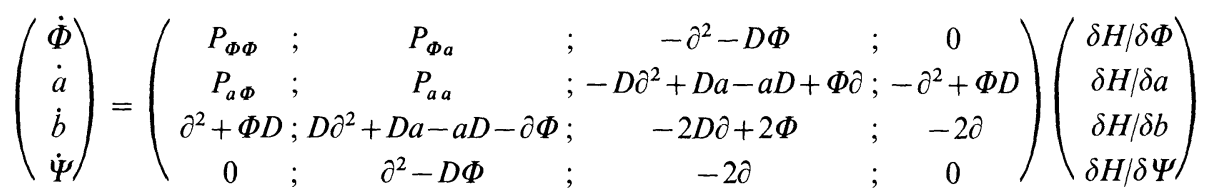


with

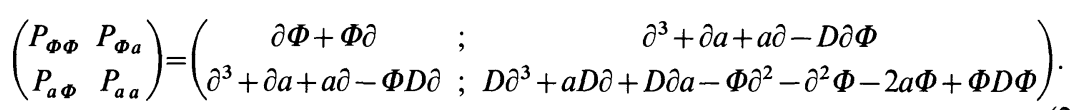

We remark that the operator in (3.22) is not a Hamiltonian operator, as it does not contain the proper dependence on the superfields $b$ and $\Psi$. Again we can invoke Dirac reduction to reduce (3.22) to the subspace spanned by $\Phi$ and $a$ imposing the constraints $b=0$ and $\Psi=0$. Inverting the lower right $2 \times 2$-block in (3.22) and using (3.17)/(3.18) one obtains the reduced Hamiltonian equation

$$
\left(\begin{array}{c}
\dot{\Phi} \\
\dot{a}
\end{array}\right)=\frac{1}{2}\left(\begin{array}{cc}
P_{\Phi \Phi}^{(\mathrm{red})} & P_{\Phi a}^{(\mathrm{red})} \\
P_{a \Phi}^{(\mathrm{red})} & P_{a a}^{(\mathrm{red})}
\end{array}\right)\left(\begin{array}{c}
\delta H / \delta \Phi \\
\delta H / \delta a
\end{array}\right)
$$

with

$$
\begin{aligned}
P_{\boldsymbol{\Phi}}^{(\mathrm{red})}= & 2 \partial \Phi+2 \Phi \partial \\
P_{\boldsymbol{\Phi}}^{\text {(red) }}= & \partial^{3}+2 \partial a+2 a \partial-D \partial \Phi-D \Phi \partial+D \Phi D \partial^{-1} \Phi, \\
P_{a \Phi}^{(\mathrm{red})}= & \partial^{3}+2 \partial a+2 a \partial-\partial \Phi D-\Phi D \partial+\Phi D \partial^{-1} \Phi D, \\
P_{a a}^{(\mathrm{red})}= & D \partial^{3}+a D \partial+D \partial a+D a \partial+\partial a D-\Phi \partial^{2}-\partial^{2} \Phi-\partial \Phi \partial \\
& -2 a \Phi-D a D \partial^{-1} \Phi-\Phi D \partial^{-1} a D+\Phi D \Phi+\partial \Phi D \partial^{-1} \Phi+\Phi D \partial^{-1} \Phi \partial \\
& -\Phi D \partial^{-1} \Phi D \partial^{-1} \Phi .
\end{aligned}
$$

We observe that for $a=0$ the operator entry $P_{a a}^{\text {(red) }}$ can formally be factorized:

$$
P_{a a}^{(\mathrm{red})}=P_{a \Phi}^{(\mathrm{red})}\left(D \partial^{2}-D \Phi D\right)^{-1} P_{\Phi a}^{(\mathrm{red})} .
$$

Hence, performing the additional reduction imposing the constraint $a=0$ leads to

$$
P_{2}^{(\mathrm{red})}=P_{\Phi \Phi}^{(\mathrm{red})}-P_{\Phi a}^{(\mathrm{red})}\left(P_{a a}^{(\mathrm{red})}\right)^{-1} P_{a \Phi}^{(\mathrm{red})}=-D \partial^{2}+2 \partial \Phi+2 \Phi \partial+D \Phi D,
$$

i.e. to the second Hamiltonian operator (1.2) of (1.1). We remark that (3.16) and (3.24)/(3.25) constitute the two Hamiltonian formulations of the integrable hierarchy of isospectral equations associated to the scattering operator $L=\partial^{2}+a-\Phi D$. Hence, as a side product of these considerations we have derived a bi-Hamiltonian hierarchy of integrable equations involving two superfunctions $a$ and $\Phi$, reducing to the hierarchy of (1.1) when putting $a=0$. The first non-trivial equation in this hierarchy reads

$$
\frac{d}{d t}\left(\begin{array}{l}
\Phi \\
a
\end{array}\right)=\left(\begin{array}{c}
\left(\Phi^{\prime \prime}+6 a \Phi-3 \Phi D \Phi\right)^{\prime} \\
\left(a^{\prime \prime}+3 a^{2}-3 \Phi D a\right)^{\prime}
\end{array}\right)=\left(\begin{array}{cc}
0 ; & \partial \\
\partial ; & D \partial-\Phi
\end{array}\right) \nabla \int\left(a \Phi-\frac{1}{2} \Phi D \Phi\right) d X
$$

decomposing into

$$
\frac{d}{d t}\left(\begin{array}{l}
u \\
\xi \\
\zeta \\
b
\end{array}\right)=\left(\begin{array}{l}
\left(u^{\prime \prime}-3 u^{2}+3 \xi \xi^{\prime}+6 b u+6 \zeta \xi\right)^{\prime} \\
\left(\xi^{\prime \prime}-3 u \xi+6 b \xi\right)^{\prime} \\
\left(\zeta^{\prime \prime}-3 u b^{\prime}+6 b \zeta+3 b^{\prime} \xi\right)^{\prime} \\
\left(b^{\prime \prime}+3 b^{2}\right)^{\prime}
\end{array}\right)
$$

when inserting $a=b+\Theta \zeta$ and $\Phi=\xi+\Theta u$. 
3.3. The Case $N=2$. The elements $L=\Sigma_{k \geqq 0}\left(b_{k}+\beta_{k} D_{1}+\gamma_{k} D_{2}+a_{k} D_{1} D_{2}\right) \partial^{k}$ in $g_{+}$ are parametrized by commuting superfunctions $a_{k}, b_{k}$ and anticommuting superfunctions $\gamma_{k}, \beta_{k}$. Given a Hamiltonian function $H(L)=H\left(\ldots, b_{k}, \beta_{k}, \gamma_{k}, a_{k}, \ldots\right)$ a convenient parametrization of the dual space $g_{-}$is given by representing the gradient $\nabla H$ as the operator

$$
\nabla H=\sum_{k \geqq 0} \partial^{-k-1}\left(D_{1} D_{2} \frac{\delta H}{\delta b_{k}}+D_{2} \frac{\delta H}{\delta \beta_{k}}-D_{1} \frac{\delta H}{\delta \gamma_{k}}+\frac{\delta H}{\delta a_{k}}\right)
$$

with commuting $\delta H / \delta a_{k}, \delta H / \delta b_{k}$ and anticommuting $\delta H / \delta \gamma_{k}, \delta H / \delta \beta_{k}$. In this parametrization the trace duality again assumes the usual euclidean form

$$
\langle\dot{L}, \nabla H\rangle=\operatorname{tr}(\dot{L} \nabla H)=\sum_{k \geqq 0} \int\left(\dot{b}_{k} \frac{\delta H}{\delta b_{k}}+\dot{\beta}_{k} \frac{\delta H}{\delta \beta_{k}}+\dot{\gamma}_{k} \frac{\delta H}{\delta \gamma_{k}}+\dot{a}_{k} \frac{\delta H}{\delta a_{k}}\right) d X .
$$

The functional derivatives $\delta / \delta b_{k}, \ldots, \delta / \delta a_{k}$ are defined by directional derivatives acting from the right as in (1.3).

To derive the "first" Hamiltonian formulations for the two integrable cases of (1.8) we again have to evaluate the first Poisson structure $P_{1}$ in (3.1) at the special points $L$ given by (1.10). As a first step we evaluate $P_{1}$ on the affine subspace given by operators of the form

$$
L=\partial^{2}+b+\beta D_{1}+\gamma D_{2}+a D_{1} D_{2}
$$

parametrized by four superfunctions $a, b, \gamma$ and $\beta$. It will turn out that this space defines a proper Poisson submanifold of $P_{1}$. According to (3.30) we insert

$$
\nabla H=\partial^{-1}\left(D_{1} D_{2} \frac{\delta H}{\delta b}+D_{2} \frac{\delta H}{\delta \beta}-D_{1} \frac{\delta H}{\delta \gamma}+\frac{\delta H}{\delta a}\right)
$$

into (3.1). The Hamiltonian equation $\dot{L}=\dot{b}+\dot{\beta} D_{1}+\dot{\gamma} D_{2}+\dot{a} D_{1} D_{2}=P_{1} \nabla H$ decomposes into

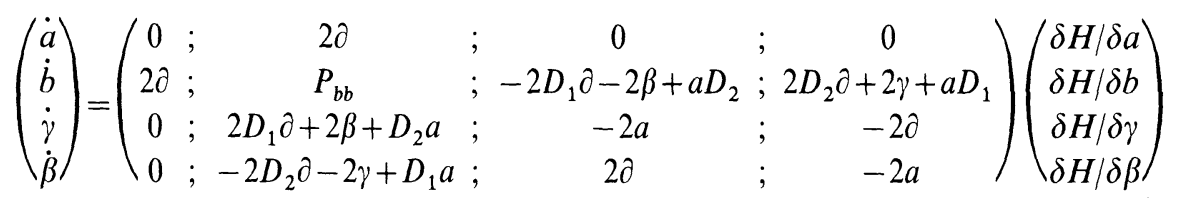

with

$$
P_{b b}=2 D_{1} D_{2} \partial-a \partial-\partial a+\beta D_{2}-D_{2} \beta-\gamma D_{1}+D_{1} \gamma .
$$

We now have to impose constraints on the coefficients $a, b, \gamma$ and $\beta$ in (3.32) to obtain the operators (1.10) from the more general (3.32). Starting with the matrix in (3.34) the corresponding Dirac reduction will yield the wanted "first" Hamiltonian structure.

The Case $\alpha=-2$. For the reduction from the general operator (3.32) to (1.10) the constraints

$$
a=2 \Phi, b=0, \gamma=\left(D_{1} \Phi\right), \beta=-\left(D_{2} \Phi\right),
$$

have to be imposed. We first perform the coordinate transformation $(a, b, \gamma, \beta) \rightarrow$ 
$(\Phi, b, \tilde{\gamma}, \tilde{\beta})$ defined by $a=2 \Phi, \gamma=\tilde{\gamma}+\left(D_{1} \Phi\right), \beta=\tilde{\beta}-\left(D_{2} \Phi\right)$, such that the constraint assume the simpler form $b=0, \tilde{\gamma}=0$ and $\tilde{\beta}=0$. The Hamiltonian equation (3.34) now translates to

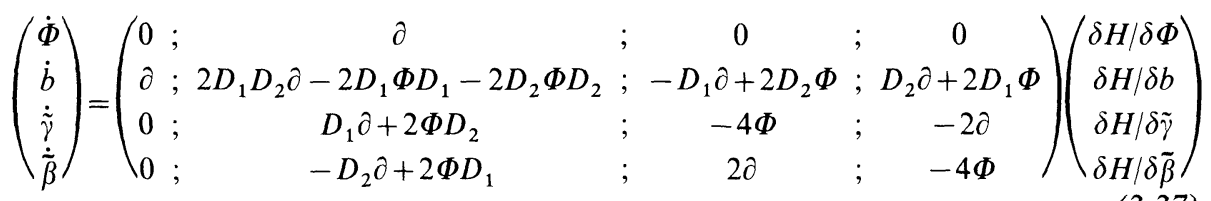

where we already have put $b=\tilde{\gamma}=\tilde{\beta}=0$. For convenience the reduction of this operator is performed in two steps. We first take into account the constraints $\tilde{\gamma}=\tilde{\beta}=0$. Using $(3.17) /(3.18)$ the Hamiltonian equation (3.37) reduces to

$$
\left(\begin{array}{l}
\dot{\Phi} \\
\dot{b}
\end{array}\right)=\left(\begin{array}{cc}
0 ; & \partial \\
\partial ; & 2 D_{1} D_{2} \partial-2 D_{1} \Phi D_{1}-2 D_{2} \Phi D_{2}-\Delta
\end{array}\right)\left(\begin{array}{l}
\delta H / \delta \Phi \\
\delta H / \delta b
\end{array}\right)
$$

where

$\Delta=\left(-D_{1} \partial+2 D_{2} \Phi ; D_{2} \partial+2 D_{1} \Phi\right)\left(\begin{array}{ccc}-4 \Phi & ; & -2 \partial \\ 2 \partial & ; & -4 \Phi\end{array}\right)^{-1}\left(\begin{array}{c}D_{1} \partial+2 \Phi D_{2} \\ -D_{2} \partial+2 \Phi D_{1}\end{array}\right)$.

Observing

$$
\left(\begin{array}{c}
D_{1} \partial+2 \Phi D_{2} \\
-D_{2} \partial+2 \Phi D_{1}
\end{array}\right)=\left(\begin{array}{ccc}
-4 \Phi & ; & -2 \partial \\
2 \partial & ; & -4 \Phi
\end{array}\right)\left(\begin{array}{c}
-\frac{1}{2} D_{2} \\
-\frac{1}{2} D_{1}
\end{array}\right)
$$

one concludes

$$
\Delta=\left(-D_{1} \partial+2 D_{2} \Phi ; D_{2} \partial+2 D_{1} \Phi\right)\left(\begin{array}{c}
-\frac{1}{2} D_{2} \\
-\frac{1}{2} D_{1}
\end{array}\right)=D_{1} D_{2} \partial-D_{1} \Phi D_{1}-D_{2} \Phi D_{2},
$$

such that (3.38) reads

$$
\left(\begin{array}{l}
\dot{\Phi} \\
\dot{b}
\end{array}\right)=\left(\begin{array}{cc}
0 ; & \partial \\
\partial ; & D_{1} D_{2} \partial-D_{1} \Phi D_{1}-D_{2} \Phi D_{2}
\end{array}\right)\left(\begin{array}{l}
\delta H / \delta \Phi \\
\delta H / \delta b
\end{array}\right) .
$$

Now the final reduction to $b=0$ leads to the "first" Hamiltonian formulation

$$
\dot{\Phi}=\partial\left(-D_{1} D_{2} \partial+D_{1} \Phi D_{1}+D_{2} \Phi D_{2}\right)^{-1} \partial \frac{\delta H}{\delta \Phi},
$$

of the integrable equations associated to (1.10), $\alpha=-2$. Again, to avoid the formal inverse in (3.43) we consider the inverse of this operator leading to the properly defined symplectic form (2.12).

The Case $\alpha=4$. For the reduction from the general operator (3.32) to (1.10) now the constraints

$$
a=-2 \Phi, b=-\left(D_{1} D_{2} \Phi\right)-\Phi^{2}, \gamma=-\left(D_{1} \Phi\right), \beta=\left(D_{2} \Phi\right)
$$

have to be imposed. We first perform the coordinate transformation $(a, b, \gamma, \beta) \rightarrow$ $(\Phi, \tilde{b}, \tilde{\gamma}, \tilde{\beta})$ defined by $a=-2 \Phi, \quad b=\tilde{b}-\left(D_{1} D_{2} \Phi\right)-\Phi^{2}, \quad \gamma=\tilde{\gamma}-\left(D_{1} \Phi\right)$, 
$\beta=\tilde{\beta}+\left(D_{2} \Phi\right)$, such that the constraints assume the simpler form $\tilde{b}=0, \tilde{\gamma}=0$ and $\widetilde{\beta}=0$. The Hamiltonian equation (3.34) now translates to

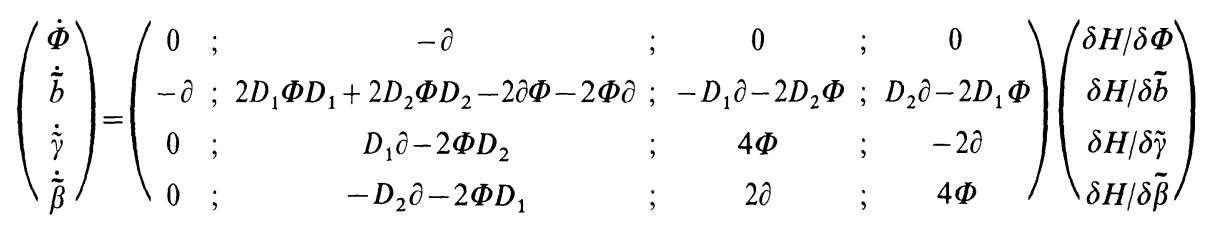

where we already have put $\tilde{b}=\tilde{\gamma}=\tilde{\beta}=0$. The reduction of this operator is performed in two steps as before. Taking into account the constraints $\tilde{\gamma}=\widetilde{\beta}=0$ the Hamiltonian equation (3.45) reduces via $(3.17) /(3.18)$ to

$$
\left(\begin{array}{c}
\dot{\Phi} \\
\tilde{b}
\end{array}\right)=\left(\begin{array}{ccc}
0 & ; & -\partial \\
-\partial & ; 2 D_{1} \Phi D_{1}+2 D_{2} \Phi D_{2}-2 \partial \Phi-2 \Phi \partial-\Delta
\end{array}\right)\left(\begin{array}{c}
\delta H / \delta \Phi \\
\delta H / \delta \tilde{b}
\end{array}\right)
$$

where

$$
\Delta=\left(-D_{1} \partial-2 D_{2} \Phi ; D_{2} \partial-2 D_{1} \Phi\right)\left(\begin{array}{cc}
4 \Phi ;-2 \partial \\
2 \partial ; 4 \Phi
\end{array}\right)^{-1}\left(\begin{array}{c}
D_{1} \partial-2 \Phi D_{2} \\
-D_{2} \partial-2 \Phi D_{1}
\end{array}\right)
$$

Observing

$$
\left(\begin{array}{c}
D_{1} \partial-2 \Phi D_{2} \\
-D_{2} \partial-2 \Phi D_{1}
\end{array}\right)=\left(\begin{array}{ccc}
4 \Phi & ; & -2 \partial \\
2 \partial & ; & 4 \Phi
\end{array}\right)\left(\begin{array}{c}
-\frac{1}{2} D_{2} \\
-\frac{1}{2} D_{1}
\end{array}\right)
$$

one concludes

$$
\Delta=\left(-D_{1} \partial-2 D_{2} \Phi ; D_{2} \partial-2 D_{1} \Phi\right)\left(\begin{array}{c}
-\frac{1}{2} D_{2} \\
-\frac{1}{2} D_{1}
\end{array}\right)=D_{1} D_{2} \partial+D_{1} \Phi D_{1}+D_{2} \Phi D_{2},
$$

such that (3.46) reads

$$
\left(\begin{array}{c}
\dot{\Phi} \\
\tilde{\tilde{b}}
\end{array}\right)=\left(\begin{array}{ccc}
0 & ; & -\partial \\
-\partial & ; & -D_{1} D_{2} \partial-2 \partial \Phi-2 \Phi \partial+D_{1} \Phi D_{1}+D_{2} \Phi D_{2}
\end{array}\right)\left(\begin{array}{c}
\delta H / \delta \Phi \\
\delta H / \delta \tilde{b}
\end{array}\right)
$$

Now the final reduction to $\tilde{b}=0$ leads to the "first" Hamiltonian formulation

$$
\dot{\Phi}=\partial\left(D_{1} D_{2} \partial+2 \partial \Phi+2 \Phi \partial-D_{1} \Phi D_{1}-D_{2} \Phi D_{2}\right)^{-1} \partial \frac{\delta H}{\delta \Phi},
$$

of the integrable equations associated to $(1.10), \alpha=4$. We observe that the formal inverse of the Hamiltonian operator $P_{2}$ defined by (1.9) turns up in this "first" Hamiltonian formulation. The Hamiltonian operator in (3.51) multiplied with the formal inverse of $P_{2}$ yields a formal recursion operator $\partial P_{2}^{-1} \partial P_{2}^{-1}$ factorizing into the square of the simpler recursion operator $R^{-1}:=\partial P_{2}^{-1}$. Applying this recursion operator to $P_{2}$ leads to $P_{1}=\partial$ as the simplest choice for the "first" Hamiltonian operator for the integrable hierarchy. From this derivation it is obvious that the recursive structure of the case $\alpha=4$ is richer than the corresponding structure for $\alpha=-2$, as the recursion operator coming from the abstract Poisson brackets (3.1) 
can be factorized in the latter case. This accounts for the "additional" symmetries in this case and reflects the fact that the Lax operator (1.10) could also be factorized for $\alpha=4$.

We remark that in this case the derivation of the bi-Hamiltonian structure associated to the Lax operator can be simplified by evaluating the abstract structures (3.1) with the simpler Lax operator $\hat{L}=(-L)^{1 / 2}=D_{1} D_{2}+\Phi$. We have checked that by a similar calculation (including suitable Dirac reductions) the first abstract Hamiltonian structure in (3.1) with $\hat{L}$ instead of $L$ directly reduces to $P_{1}=\partial$, whereas the abstract $P_{2}$ in (3.1) reduces to the Poisson tensor (1.9).

\section{Conclusions}

We have exhibited the bi-Hamiltonian nature of the supersymmetric invariant cases of integrable $\mathrm{KdV}$-extensions related the the $N=1$ and $N=2$ conformal super algebras. For two cases inverse Hamiltonian formulations in terms of (pre-) symplectic operators had to be taken into account. The third case shows the peculiar phenomenon of admitting "additional" invariants originating from a factorization of the corresponding Lax operator. In any case we obtain a hereditary recursion operator, a set of master symmetries and hence a recursive scheme for a simple construction of the higher symmetries and conservation laws for these systems.

All structures can be calculated in a straightforward way from a general scheme on pseudo-differential operators. We have demonstrated that this scheme yields a straightforward way to calculate the Poisson brackets for the integrable equations from the associated scattering problem. The fact that some of the examples admit inverse Hamiltonian structures involving symplectic forms instead of Poisson operators is "explained" by the reduction properties of the abstract brackets (3.1). The scheme suggests a systematic way of looking for further bi-Hamiltonian super-extensions of $\mathrm{KdV}$-like equations by evaluating the abstract equations (3.2) with their abstract Hamiltonian operators (3.1) for a larger class of Lax operators and/or other choices for the abstract algebra $g$ in Sect. 3. E.g., the bi-Hamiltonian structure (3.16) and (3.24)/(3.25) of the isospectral equations associated to the scattering operator $L=\partial^{2}+a-\Phi D$ was easily derived leading to further integrable extensions of the $\mathrm{KdV}$ such as (3.28).

\section{References}

1. Belavin, A., et al.,: Nucl. Phys. B 24, 333 (1984)

2. Friedan, D., et al.,: Phys. Rev. Lett. 52, 1575 (1984)

3. Kupershmidt, B.: Phys. Lett. A 109, 417 (1985)

4. Yamanaki, I., Sasaki, R.: Prog. Theor. Phys. 79, 1167 (1988)

5. Manin, Y., Radul, A.: Commun. Math. Phys. 98, 65 (1985)

6. Gervais, J. L.: Phys. Lett. 160B, 277 (1985)

7. Chaichian, M., Lukierski, J.: Phys. Lett. 212B, 461 (1988)

8. Khovanova, T.: Teor. Math. Phys. 72 (2), 306 (1987) (in Russian)

9. Mathieu, P.: J. Math. Phys. 29, 2499 (1988)

10. Laberge, C., Mathieu, P.: Phys. Lett. 215B, 718 (1988)

11. Fuchssteiner, B., Fokas, A. S.: Physica 4D, 47 (1981) 
12. Fuchssteiner, B.: Progr. Theor. Phys. 70, 1508 (1983)

13. Oevel, W.: In 'Topics in soliton theory and exactly solvable nonlinear equations.' Ablowitz, M., Fuchssteiner, B., Kruskal, M. (eds.) Singapore; World Scientific (1987), 108

14. Oevel, W.: Master symmetries: Weak action/angle structure for Hamiltonian and nonHamiltonian systems, preprint, Paderborn (1986)

15. Fuchssteiner, B.: Progr. Theor. Phys. 68, 1082 (1982)

16. Fuchssteiner, B., Oevel, W.: J. Math. Phys. 23, 358 (1982)

17. Oevel, W.: Phys. Lett. 94A, 404 (1983)

18. Kupershmidt, B.: unpublished; reported in: Labelle, P., Mathieu, P.: A new $\mathrm{N}=2$ supersymmetric KdV equation, Laval preprint (1990)

19. Gelfand, I. M., Dikii, L. A.: Funct. Anal. Appl. 10, 259 (1976)

20. Adler, M.: Invent. Math. 50, 219 (1979)

21. Semenov-Tian Shansky, M. A.: Funct. Anal. Appl. 17, 259 (1983)

22. Oevel, W.: J. Math. Phy. 30, 1140 (1989)

23. Oevel, W., Ragnisco, O.: Physica A 161, 181 (1989)

24. Kupershmidt, B.: Lett. Math. Phys. 9, 323 (1985)

Communicated by $\mathrm{H}$. Araki 